\title{
ZLIB++: Object-Oriented Numerical Library for Differential Algebra
}

\author{
N. Malitsky and A. Reshetov \\ Superconducting Super Collider Laboratory* \\ 2550 Beckleymeade Avenue \\ Dallas, TX 75237 \\ and \\ Y. Yan \\ Stanford Linear Accelerator Center \\ Stanford, CA 94309
}

January 1994

* Operated by the Universities Research Association, Inc., for the U.S. Department of Energy under Contract No. DE-AC35-89ER40486. 


\subsection{INTRODUCTION}

New software engineering tools and object-oriented design have a great impact on the software development process. But in high energy physics all major packages were implemented in FORTRAN and porting of these codes to another language is rather complicated, primarily because of their huge size and heavy use of FORTRAN mathematical libraries. But some intrinsic accelerator concepts, such as nested structure of modern accelerators, look very pretty when implemented with the object-oriented approach. In this paper we present the object-oriented version of ZLIB,${ }^{1}$ numerical library for differential algebra, ${ }^{2}$ and show how the modern approaches can simplify the development and support of accelerator codes, decrease code size, and allow description of complex mathematical transformations by simple language.

\subsection{TRUNCATED POWER SERIES}

The truncated power series (TPS) expansion of the arbitrary function $U(\overrightarrow{\mathbf{z}})$ is defined as: 1

$$
U(\overrightarrow{\mathbf{z}})=\sum_{k=0}^{\Omega} u(\overrightarrow{\mathbf{k}}) \overrightarrow{\mathbf{z}}^{\overrightarrow{\mathbf{k}}}
$$

where

$$
\begin{aligned}
\overrightarrow{\mathbf{z}}^{T} & =\left[z_{1}, z_{2}, \ldots, z_{n}\right], \\
\overrightarrow{\mathbf{z}}^{\overrightarrow{\mathbf{k}}} & \equiv z_{1}^{k_{1}} z_{2}^{k_{2}} \ldots z_{n}^{k_{n}}, \\
k & =\sum_{i=1}^{n} k_{i}, \text { for } 0 \leq k_{i} \leq \Omega .
\end{aligned}
$$

In the new object-oriented version of ZLIB, TPS is considered as $\mathrm{C}++$ class ZSeries, which includes overloaded assignment, additive, multiplicative operators and two additional functions dif and poisson (Appendix A). To simplify the form of the TPS transformation equations we include the additional private member order [order $\Omega$ of TPS expansion in Eq. (1)] and several rules for its usage. Below we describe the main elements of class ZSeries and their relationship with the subroutines of the previous FORTRAN version of ZLIB. Full description of fundamental TPS operations may be found in a users guide for ZLIB 1.0.1

\subsection{Definitions}

In expressions and examples in this report we will use the following notation:
$\mathbf{U}, \mathbf{V}, \mathbf{W}$
are the instances of class ZSeries;
c
is constant or variable of the double type; 


\section{ZLIB_ORDER is global variable, which determines the maximum TPS order; \\ ZLIB_DIM is global variable, which determines the phase-space dimension.}

The parameters ZLIB_ORDER and ZLIB_DIM must be defined by user before all assignment operators, because they determine memory allocation for members of class ZSeries.

\section{$2.2 \quad$ Assignment 0perators}

An assignment operator is used to set a ZSeries variable to constant or another ZSeries value. This operator returns void and so a vague statement $(\mathbf{a}=\mathbf{b})=\mathbf{c}$ will result in a syntax error. Here is the summary of different cases of assignment operator:

2.2.1 Operator $=$

1. $\mathbf{W}=\mathbf{c}$;

$$
\begin{array}{ll}
w(\overrightarrow{\mathbf{k}})=\mathbf{c} & \text { for } 0 \leq k \leq \mathbf{W} \text {.order } \\
& \text { W.order }=0 .
\end{array}
$$

2. $\mathbf{W}=\mathbf{U}$;

$$
\begin{array}{ll}
w(\overrightarrow{\mathbf{k}})=u(\overrightarrow{\mathbf{k}}) \quad \text { for } 0 \leq k \leq \mathbf{U} . \text { order } \\
& \text { W.order }=\mathbf{U} . \text { order }
\end{array}
$$

2.2.2 Operators $t=$ and $-=$

1. $\mathbf{W}+=\mathbf{c}$;

$$
\begin{array}{ll}
w(\overrightarrow{\mathbf{k}})=w(\overrightarrow{\mathbf{k}})+\mathbf{c} & \text { for } k=0, \\
w(\overrightarrow{\mathbf{k}})=w(\overrightarrow{\mathbf{k}}) & \text { for } 0<k \leq \mathbf{W . o r d e r} .
\end{array}
$$

2. $\mathbf{W}+=\mathbf{U}$;

$$
\begin{gathered}
W(\overrightarrow{\mathbf{z}})=\sum_{k=0}^{\mathbf{W} . \text { order }} w(\overrightarrow{\mathbf{k}}) \overrightarrow{\mathbf{z}}^{\overrightarrow{\mathbf{k}}}+\sum_{k=0}^{\text {U.order }} u(\overrightarrow{\mathbf{k}}) \overrightarrow{\mathbf{z}}^{\mathbf{k}}, \\
\text { W.order }=\max (\mathbf{W} \text {.order, U.order }) .
\end{gathered}
$$

3. $\mathbf{W}-=\mathbf{c}$; and $\mathbf{W}-=\mathbf{U}$;

These subtraction operators are determined similar to addition (Section 2.2.2).

\subsubsection{Operator $*=$}

1. $\mathbf{W}^{*}=\mathbf{c}$;

$$
w(\overrightarrow{\mathbf{k}})=w(\overrightarrow{\mathbf{k}}) * \mathbf{c} \quad \text { for } 0 \leq k \leq \mathbf{W} \text {.order } .
$$


2. $\mathbf{W}^{*}=\mathbf{U}$;

$$
W(\overrightarrow{\mathbf{z}})=\sum_{k=0}^{\text {W.order }} w(\overrightarrow{\mathbf{k}}) \overrightarrow{\mathbf{z}}^{\mathbf{k}} * \sum_{k=0}^{\text {U.order }} u(\overrightarrow{\mathbf{k}}) \overrightarrow{\mathbf{z}} \overrightarrow{\mathbf{k}}^{\overrightarrow{\mathbf{k}}},
$$

W.order $=\min (\mathbf{W}$. order + U.order.ZLIB_ORDER $)$.

\subsubsection{Operator $/=$}

1. $\mathbf{W} /=\mathbf{c}$;

$$
w(\overrightarrow{\mathbf{k}})=w(\overrightarrow{\mathbf{k}}) / \mathbf{c} \quad \text { for } 0 \leq k \leq \mathbf{W} . \text { order } .
$$

2. $\mathbf{W} /=\mathbf{U}$;

The implementation of this operator is based on two functions: multiplication (Section 2.2.3) and inversion $1 / \mathbf{U}$ (Section 2.4) and defined as:

$$
\mathbf{W} *=(1 / \mathrm{U})
$$

\subsection{Additive and Multiplicative Operators}

Additive ( + and - ) and multiplicative (* and /) operators are similar to corresponding assignment operators (Sections 2.2.2, 2.2.3 and 2.2.4), but unlike assignment operators they return reference to tmpZSeries, temporary instance of class ZSeries, which is created as a result of the expression. This allows user to write usual mathematical expressions as:

$$
\mathbf{W} *=(\mathbf{U}-\mathbf{c}) / \mathbf{V} * \mathbf{c}+\ldots
$$

\subsection{Inverse Operator}

Inversion of the object $\mathbf{U}$ is expressed as a Taylor expansion of $1 /(1+\mathbf{V})$, where $\mathbf{V}=$ $(\mathbf{U}-u(0)) / u(0)$ :

$$
\begin{aligned}
\mathbf{W}=1 . / \mathbf{U} & =\frac{1}{u(0)\left(1+\frac{\mathrm{U}-u(0)}{u(0)}\right)} \\
& =\frac{1}{u(0)} * \sum_{n=0}^{\mathbf{U} \cdot \operatorname{tmpOder}_{\mathbf{P}}}(-1)^{n}\left(\frac{\mathrm{U}-u(0)}{u(0)}\right)^{n}
\end{aligned}
$$

The implementation of this expression is based on ZSeries multiplicative and additive operators (Appendix B). 


\subsection{Functions}

This object-oriented version of ZLIB supports two functions dif and poisson. As in the case of additive and multiplicative operators (Section 2.3), these functions return reference to temporary object tmpZSeries and may be combined with other operators in the complex expression.

\subsubsection{Derivative}

The function $\operatorname{dif}(\mathbf{U}, i v)$ returns the partial derivative $\left(\partial / \partial z_{i v}\right) U(\overrightarrow{\mathbf{z}})$.

\subsubsection{Poisson Bracket}

Poisson bracket is the main operator of differential algebra. It associated with a Lie operator : $U(\overrightarrow{\mathbf{z}})$ : as in Reference 2 :

$$
\operatorname{poisson}(\mathbf{U}, \mathbf{V}) \equiv: U(\overrightarrow{\mathbf{z}}): V(\overrightarrow{\mathbf{z}}) \equiv[U(\overrightarrow{\mathbf{z}}), V(\overrightarrow{\mathbf{z}})]=-\left(\frac{\partial U(\overrightarrow{\mathbf{z}})}{\partial \overrightarrow{\mathbf{z}}}\right)^{T} S\left(\frac{\partial V(\overrightarrow{\mathbf{z}})}{\partial \overrightarrow{\mathbf{z}}}\right)
$$

where $\mathrm{S}$ is the symplectic identity.

\subsection{Access 0perators}

Access operators may be used to change directly the private members of class ZSeries (coefficients of the TPS and its order order) and some specific rules of the transformation of order, described in Section 2.3. These rules may be generalized and expressed as the following:*

Rule 1 The order of the object $\mathbf{W}$, created by constructor ZSeries :: ZSeries(), is equal to zero, i.e., for the new object $\mathbf{W}$.order $=0$;

Rule 2 The order of the object $\mathbf{W}$ in the left side of an assignment operator $\mathbf{W}=\mathbf{U}$ is determined by the order of $\mathbf{U}$, i.e., $\mathbf{W}$.order $=\mathbf{U}$.order.

Rule 3 The order of the object $\mathbf{W}$, which is obtained as a result of additive operations $(+=,-=,+$ and -$)$ is equal to the maximum order of items $\mathbf{U}$ and $\mathbf{V}$, i.e., W.order = $\max ($ U.order, V.order).

Rule 4 The order of the object $\mathbf{W}$, which is obtained as a result of multiplicative operations $(*=, /=, *$ and /) or binary functions (poisson( $(\mathbf{U}, \mathbf{V})$ ) is equal to $\min (\mathbf{U}$.order + V.order, ZLIB_ORDER).

\footnotetext{
* These rules are valid also for the expressions with the constant c, regarding its order to be zero.
} 


\subsubsection{Operator()(int)}

This operator (round brackets) allows user to temporarily change the order W.order of the object $\mathbf{W}$ in the intermediate expression. To assign new order newOrder "permanently" user could use the following construction:

$$
\mathbf{U}=\mathbf{U}(\text { newOrder }) \text {. }
$$

\subsubsection{Operator()(int, int)}

The second parameter in operator() allows user to bypass Rule 4 for multiplicative operators (Section 2.6) in accordance with the following definition:

$$
\begin{aligned}
\mathbf{W} & =\mathbf{U} * \mathbf{V}(\mathbf{V} \text {.order, }, \mathbf{m} \cdot \text { mltOrder }) \\
\mathbf{W} . \text { order } & =\min (\mathbf{V} \cdot \mathbf{m l t O} \text { Order }, \mathbf{W} \cdot \mathbf{m l t O r d e r}(\text { from Rule } 4)) .
\end{aligned}
$$

We used operator( $)$ in the implementation of the inverse operator for the order of $1 / \mathrm{U}$ not to exceed the order of $\mathrm{U}$ (Section 2.4 and Appendix B).

\subsubsection{Operator[ ]}

The subscripting operator[ ] (int $\mathrm{i})$ of the object $\mathbf{U}$ returns the reference to its private member U.z[i], which represents the i-th coefficient $u(i)$ of the TPS expansion in Eq. (1).

\subsection{MAPS}

Map $\overrightarrow{\mathbf{U}}(\overrightarrow{\mathbf{z}})$ is the development of concept of the truncated power series (TPS) and defined as the m-dimensional vector of TPS expansions, Eq. (1):

$$
\overrightarrow{\mathbf{U}}(\overrightarrow{\mathbf{z}})=\sum_{k=0}^{\Omega} \overrightarrow{\mathbf{u}}(\overrightarrow{\mathbf{k}}) \overrightarrow{\mathbf{z}}^{\overrightarrow{\mathbf{k}}},
$$

where

$$
\begin{aligned}
\overrightarrow{\mathbf{u}}(\overrightarrow{\mathbf{k}})^{T} & =\left[u_{1}(\overrightarrow{\mathbf{k}}), u_{2}(\overrightarrow{\mathbf{k}}), \ldots, u_{m}(\overrightarrow{\mathbf{k}})\right], \\
k & =\sum_{i=1}^{n} k_{i}, \text { for } 0 \leq k_{i} \leq \Omega .
\end{aligned}
$$

In the object-oriented version of ZLIB the map is considered as an object of $\mathrm{C}++$ class ZMap, which naturally was derived from the class ZSeries (Appendix C). Moreover, all arithmetical operators of class Zmap follow the same Rules (Section 2.6) and are based on ZSeries multiplicative and additive operators. This leads to similar implementation of member functions for these two classes. For example, compare inverse operator for ZMap (Appendix D) and ZSeries (Appendix B). Below we describe only the essential distinction between these classes and the additional ZMap functions. 


\subsection{Unit Map}

Unlike the truncated power series the unit map $I$ is defined as a vector:

1. $\mathbf{I}=1$;

$$
\begin{aligned}
\overrightarrow{\mathbf{I}}(\overrightarrow{\mathbf{z}}) & =\overrightarrow{\mathbf{z}}, \\
\text { I.order } & =1 .
\end{aligned}
$$

\subsection{Operator[ ]}

The subscripting operator [ ] (int i) of the object $\mathbf{M}$ (the instance of class ZMap) returns the reference to its private member M.z[i] the instance of class ZSeries) which represents the $\mathrm{i}$-th member $u_{i}$ of the m-dimensional vector $\overrightarrow{\mathbf{u}}$ in Eq. (2).

\subsection{Function Poisson (ZSeries\&, ZMap\&)}

Function poisson(V, M) returns the Poisson bracket: $V(\overrightarrow{\mathbf{z}}): \overrightarrow{\mathbf{M}}(\overrightarrow{\mathbf{z}}) \equiv[V(\overrightarrow{\mathbf{z}}), \overrightarrow{\mathbf{M}}(\overrightarrow{\mathbf{z}})]^{2}$ Its implementation is based on the similar ZSeries function as:

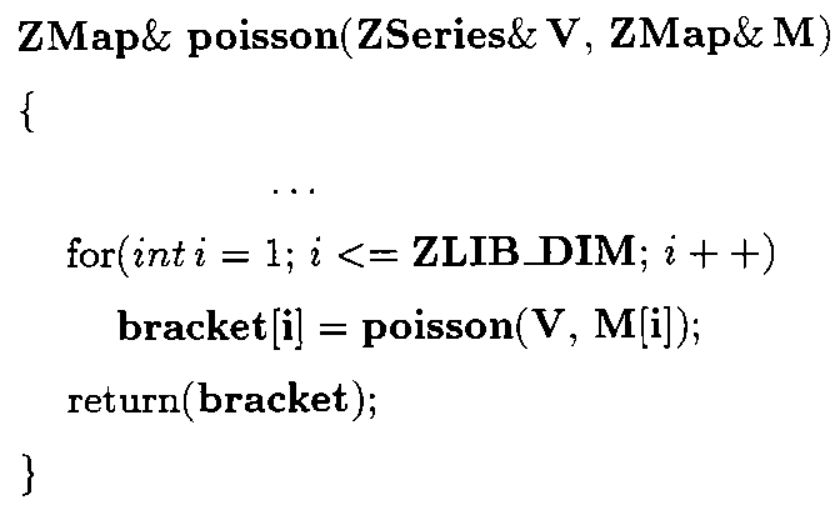

where bracket is the temporary instance of class ZMap.

\subsection{TRACKING}

Tracking is one of the most important procedures in accelerator codes. In our "language" it is defined simply and naturally:

$$
\mathbf{y}=\mathbf{M} * \mathbf{x}
$$

where $\mathbf{M}$ is the $\mathbf{Z M a p}$ object, and $\mathbf{x}$ and $\mathbf{y}$, the instances of class Particle (Appendix D), consist of the particle coordinates correspondingly before and after one turn. For multiparticle tracking user may use usual $\mathrm{C}++$ operators: 
$\operatorname{main}()$

\{

\section{ZMap M;}

Particle ** $\mathbf{x}$;

for $($ int $i=1 ; i<=$ numberParticles $; i++)$

for (int $j=0 ; j<$ numberTurns; $j++$ )

$\mathbf{x}[i][j+1]=\mathbf{M} * \mathbf{x}[i][j] ;$

\}

\subsection{CONCLUSION}

In this report we have described a new object-oriented version of the ZLIB package. ZLIB ++ defines two classes (ZSeries and ZMap) to represent specific accelerator objects. We argued that simple $\mathrm{C}$ operators ( such as $=,+,-, /, *$ ) are naturally suited to implement different mathematical algorithms with these objects. It enables one to write simple, selfdocumented programs for applications of numerical methods of differential algebra in high energy physics.

ZLIB ++ was designed to be a foundation for further developments. New classes could be naturally derived from the base ones, enabling expansion of the package in the open architecture style. 


\section{ACKNOWLEDGEMENTS}

We would like to thank Dr. G. Bourianoff for his strong support and for various helpful discussions. 


\section{REFERENCES}

1. Y. Yan and Chiung-Ying Yan, "ZLIB-A Numerical Library for Differential Algebra," SSC Laboratory Report SSCL-300, (1990).

2. Y. Yan, "Applications of Differential Algebra to Single-Particle Dynamics in Storage Rings," SSC Laboratory Report SSCL-500, (1991). 


\section{APPENDIX A}

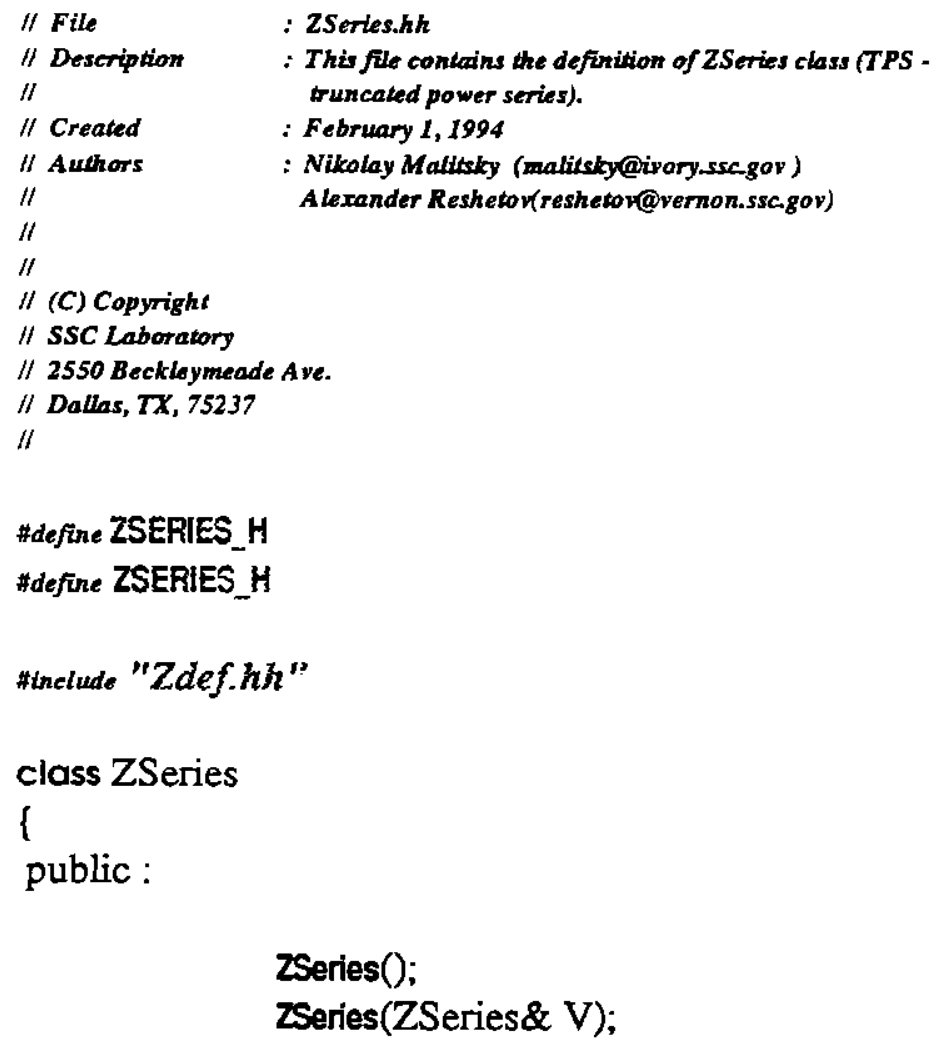

// Access operators \& function

ZSeries\& operator()(int i1);

ZSeries\& operator()(int i1, int i2); double\& operatorD(Int number);

/ Assignment operators

void operator $=$ (double c); void operator $=(Z$ Series\& V); void operator $+=(Z$ Series\& $V)$; void operatort=(double c); void operator- $=(Z$ Series\& V); void operator $=$ (double $c)$; void operator* $=(Z$ Series\& V); void operator* ${ }^{*}$ (double $\mathrm{c}$ ); void operator/=(ZSeries\& V); void operator/=(double c);

ZSeries\& operator+(ZSeries\& V); 
ZSeries\& operator-(ZSeries\& V):

ZSeries\& operator*(ZSeries\& V);

ZSeries\& operator/(ZSeries\& V);

// Friend operalors

friend ZSeries\& operator-(ZSeries\& V);

friend ZSeries\& operator+(ZSeries\& V, double $c)$;

friend ZSeries\& operator+(double c, ZSeries\& V);

trlend ZSeries\& operator-(ZSeries\& V, double c);

triend ZSeries\& operator-(double c, ZSeries\& V);

triend ZSeries\& operator*(ZSeries\& V, double c);

friend ZSeries\& operator* (doubie c, ZSeries\& V);

friend ZSeries\& operator/(ZSeries\& V, double c);

friend ZSeries\& operator/(double c, ZSeries\& V);

friend int zSeriesSlze(ZSeries\& V);

triend ostream\& operator $<<$ (ostream\& out, ZSeries\& V);

//Funetions

friend ZSeries\& dit(ZSeries\& V, int iv);

triend ZSeries\& poisson(ZSeries\& V1, ZSeries\& V/2);

ZSeries();

private:

\};

\#endy 


\section{APPENDIX B}

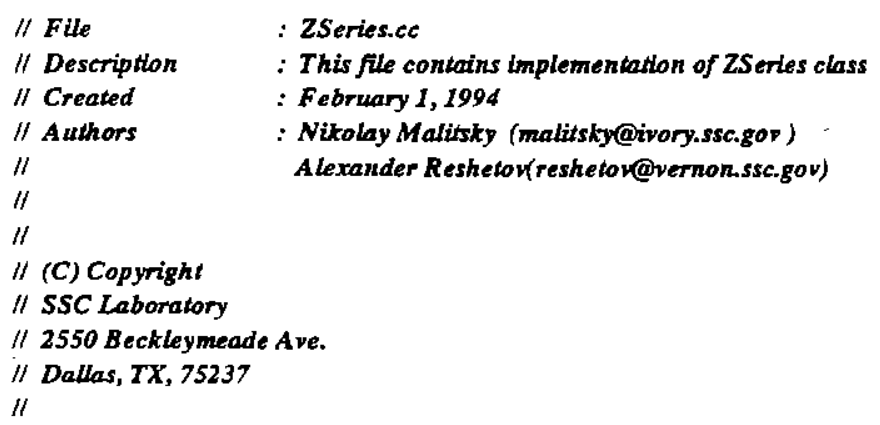

ZSeries\& operator/(double c, ZSeries\& V)

\{

ZSeries El;

ZSeries sum;

$\mathrm{El}=\mathrm{V}$;

Int $\mathrm{tN}=$ El.prepareTmpzSeries();

iff (tabs $(\mathrm{El}[1])<\mathrm{ZTNNY})$

\{

cerr $<<$ "Error: ZSeries:Binary operator c/V : fabs $(V[1])=$; cerr $<$ tabs(El[1]) $<$ " < " $<$ ZTINY $<$ " Vn";

exit(1);

\}

double linearInv = $1 / \mathrm{El}[1]$;

sum = linearInv;

$\mathrm{El}-=\mathrm{El}[1]$

$\mathrm{E} 1 *=-1$;

int now $=$ ZSeriesOrder $(E \mathrm{El})$;

for (int $i=1 ; i<=$ now; $i++$ )

\{

sum $*=E l(i, i)$;

sum $+=1$;

sum $*=$ linearInv;

\}

$\operatorname{sum} *=\mathrm{c}$;

*sum.tmpZSeries[tN] = sum; 
return (*sum.tmpZSeries[tN]);

\} 


\section{APPENDIX C}

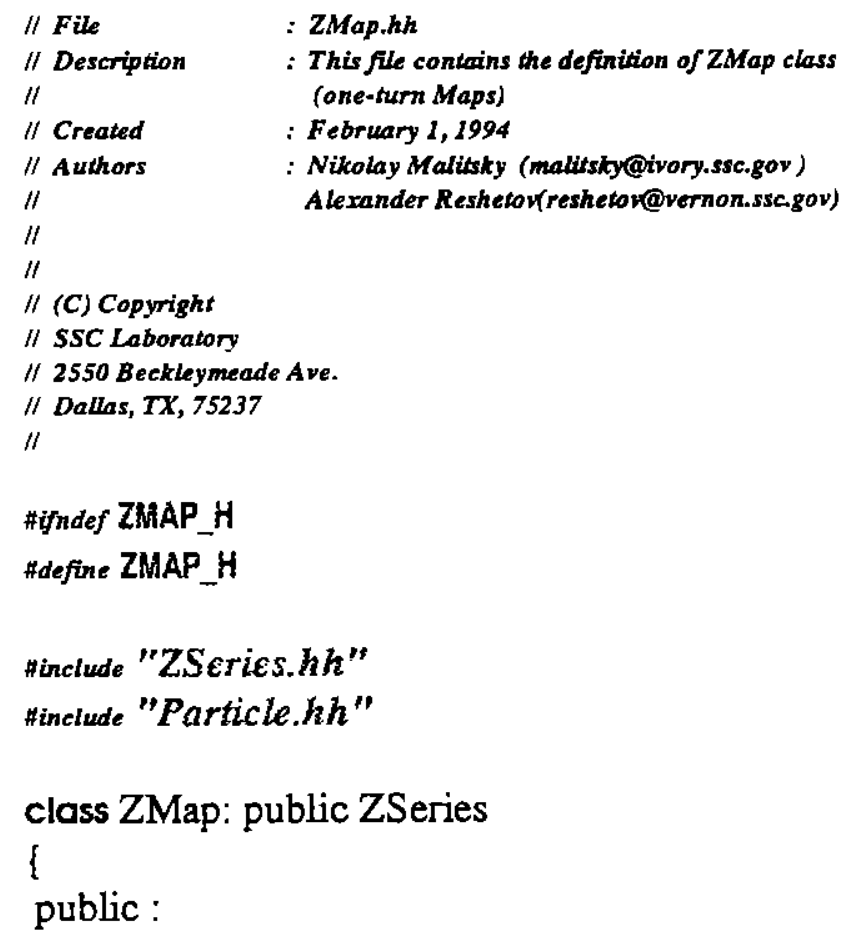

// Aceess operators \& function

ZMap\& operator()(Int in 1);

ZMap\& operator()(int in 1, int in2);

ZSeries\& operator[](int number);

/ Assignment operators

$$
\begin{aligned}
& \text { void operator }=(\text { double } \mathrm{c}) ; \\
& \text { void operator }=(\text { ZMap\& } \mathrm{M}) ; \\
& \text { void operator }=(\text { ZMap\& } \mathrm{M}) \text {; } \\
& \text { vold operator }=(\text { double } \mathrm{c}) ; \\
& \text { void operator }=(\text { ZMap\& } \mathrm{M}) ; \\
& \text { void operator }=(\text { double } \mathrm{c}) ; \\
& \text { vold operator*=(ZMap\& } \mathrm{M}) ; \\
& \text { void operator } *=(\text { double } \mathrm{c}) ; \\
& \text { void operator }=(\text { ZMap\& } \mathrm{M}) ; \\
& \text { void operator }=(\text { double } \mathrm{c}) ;
\end{aligned}
$$


ZMap\& operator+(ZMap\& M);

ZMap\& operator-(ZMap\& M);

ZMap\& operator*(ZMap\& M);

ZMap\& operator/(ZMap\& M);

// Friend operators \& function

friend ZMap\& operator-(ZMap\& M);

friend ZMap\& operator+(ZMap\& M, double c);

friend ZMap\& operator+(double c, ZMap\& M);

friend ZMap\& operator-(ZMap\& M, double c);

friend ZMap\& operator-(double c, ZMap\& M);

friend ZMap\& operator*(ZMap\& M, double c);

friend ZMap\& operator*(double c, ZMap\& M);

friend ZMap\& operator/(ZMap\& M, double c);

friend ZMap\& operator/(double c, ZMap\& M);

friend int ZMapSize(ZMap\& M);

triend ostream\& operator $<<$ (ostream\& out, ZMap\& M);

// Tracking

Particle\& operator*(Particle\& P);

// Functions

friend ZMap\& $\quad$ poisson(ZSeries\& V, ZMap\& M);

-ZMap();

private:

\}

\#endif 


\section{APPENDIX D}

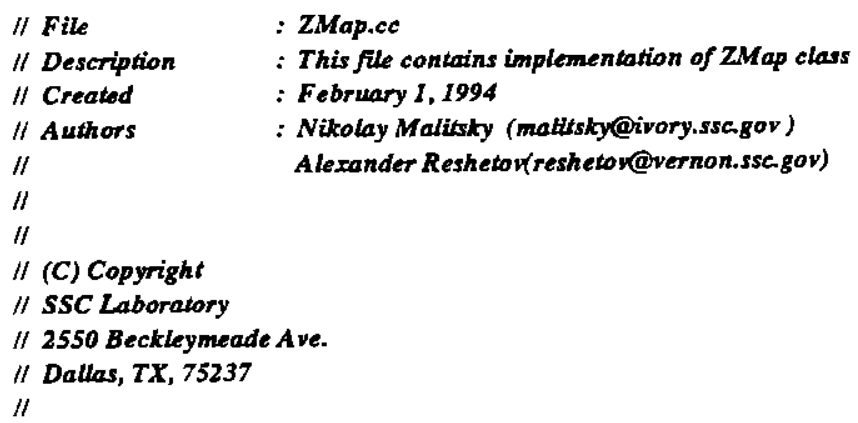

ZMap\& operator/(double c, ZMap\& M)

\{

ZMap El;

ZMap sum;

$\mathrm{El}=\mathrm{M}$;

Inf $\mathrm{tN}=$ El.prepareTmpZMap $($;

for (Int $i=1 ; i<=$ ZLBB_DM; i++)

\{

if $(\operatorname{fabs}(E][i][1])>$ TnNY)

\{

cerr « "Error: ZMap:Binary operator $\mathbf{M}$ M: ";

cerr $<<$ "labs(M.z[" $<\mathrm{i}<<"][1]=$ ";

cerr $\ll$ fabs(M.z[i][1]) $<">$ " $<$ ZTINY $<$ " Vn"; exit(1);

\}

\}

ZMap linearInv;

linearInv.lineartmeersion $(\mathrm{EI})$;

sum = linearInv;

$\mathrm{El}-=\mathrm{El}(1)$

$\mathrm{El} *=-1$;

int now = ZMapOrder $(\mathrm{El})$;

Eor $(i=2 ; i<=$ now; $i++)$

\{

sum $*=\mathrm{El}(\mathrm{i}, \mathrm{i})$;

sum $+=1$;

sum *= linearInv; 
sum ${ }^{*}=\mathrm{c}$;

*sum.tmpZMap [tN] = sum; return (*sum.tmpZMap[tN]);

\}

. 


\section{APPENDIX E}

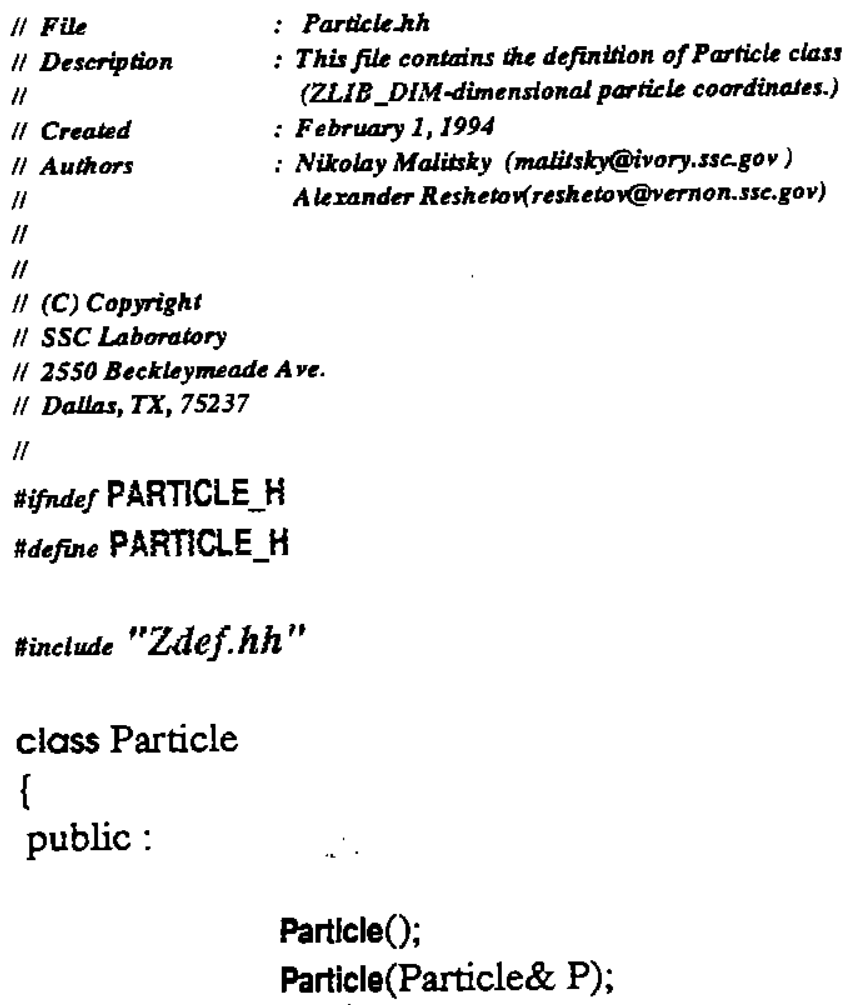

friend ostream\& operator $<<$ (ostream\& out, Particle\& P);

Particle();

private:

double* $\mathrm{z} ; \quad$ //ZIB_DIM-dimensional particle coordinates.

\};

\#endif 